\title{
Clinical features and treatment of 140 cases of Marjolin's ulcer at a major burn center in southwest China
}

\author{
FEI XIANG, HUA-PEI SONG and YUE-SHENG HUANG
}

\begin{abstract}
State Key Laboratory of Trauma, Burns and Combined Injury, Institute of Burn Research, Southwest Hospital, Third Military Medical University (Army Medical University), Chongqing 400038, P.R. China
\end{abstract}

Received August 14, 2018; Accepted February 5, 2019

DOI: $10.3892 /$ etm.2019.7364

\begin{abstract}
Marjolin's ulcer is a type of malignant tumor that occurs in scar tissue. The present study aimed to summarize and analyze the aetiology, clinical characteristics, treatment methods, metastasis and prognosis of this disease. A total of 140 cases of Marjolin's ulcer encountered at the Institute of Burn Research, Southwest Hospital (Chongqing, China) between January 2013 and December 2017 were retrospectively analyzed. Demographic data, clinical characteristics, occurrence of bone invasion and lymph node metastasis, as well as treatment and prognosis were statistically analyzed. Among the 140 patients, the initial injury or primary disease occurred at 1-75 years of age, while Marjolin's ulcer occurred at $15-85$ years of age (mean, $53.3 \pm 1.2$ years). The mean latency period was $28.8 \pm 1.7$ years. The most common initial injury of the patients was flame burns, followed by skin masses, trauma, skin ulcerations caused by repeated scratching/friction, and scalding. The age at onset of initial injury or disease in patients had a significantly negative correlation with the latency period $(\mathrm{P}<0.01)$. The most common lesion locations were the lower limbs (42.1\%), followed by the head, face and neck (34.5\%). Of the 140 patients, 46 cases $(32.9 \%)$ had bone invasion, 33 cases $(23.6 \%)$ had lymph node enlargement and only 5 cases $(3.6 \%)$ had lymph node metastasis. The skull was the bone that was most susceptible to Marjolin's ulcer invasion. The prevalence of bone invasion in patients with head, face and neck lesions was significantly higher than that in patients with lesions in other locations $(\mathrm{P}<0.01)$. The surgical methods applied were skin grafting, local flap repair, amputation and island flap repair. In the 65 cases who underwent follow-up, recurrence mainly occurred within 1 year after surgery. In conclusion, Marjolin's ulcer mainly occurred in males and was
\end{abstract}

Correspondence to: Professor Yue-Sheng Huang, State Key Laboratory of Trauma, Burns and Combined Injury, Institute of Burn Research, Southwest Hospital, Third Military Medical University (Army Medical University), 30 Gaotanyan Street, Chongqing 400038, P.R. China

E-mail: xftmmu@aliyun.com

Key words: Marjolin's ulcer, clinical features, outcome, burn, scar a scar carcinoma after a flame burn in most cases. Autologous skin grafting and local skin flap repair were the major repair methods. The peak period of recurrence was within one year after surgery and patients should receive regular follow-ups.

\section{Introduction}

Scar carcinoma is also known as Marjolin's ulcer, named after the French surgeon Marjolin who first described a tumor arising from burn scars in 1928 (1). Initially, the term 'Marjolin's ulcer' specifically referred to squamous cell carcinoma that developed after the malignant transformation of ulcers after a burn. However, as a result of in-depth study of the malignant transformation of other cellular components in scars after burns and case reports on the malignant transformation of scars caused by non-burn wounds, the current concept of Marjolin's ulcer encompasses all malignant tumors that primarily occur in body surface ulcers $(1,2)$. In terms of histopathological classification, Marjolin's ulcers now are classified into squamous cell carcinoma, basal cell carcinoma, melanoma, sarcoma and other rare cell types $(2,3)$. The majority of Marjolin's ulcers are squamous cell carcinomas, followed by basal cell carcinoma and melanoma (1). The malignant transformation rate was reported as $1-2 \%$ (4).

In general, Marjolin's ulcer remains the most common type of malignancy occurring in deep partial-thickness or full-thickness burn wounds that do not receive any surgical treatment. Other causes include pressure sores, venous ulcers, chronic osteomyelitis, unhealed wounds resulting from various causes and scars that have undergone repeated ulceration (5). The disease course of Marjolin's ulcer is long and is usually accompanied by ulcer formation. The typical Marjolin's ulcer is easy to diagnose based on a history of unhealed scar ulcers and characteristic local clinical presentations $(6,7)$. However, atypical cases are easily ignored initially or misdiagnosed as general scar ulcers. At present, the most effective treatment method for Marjolin's ulcer is surgery. Radiotherapy is recommended for patients who cannot undergo surgery or is used for consolidation treatment after surgery. Chemotherapy is not generally considered an effective therapeutic method for Marjolin's ulcer. In addition, the exact pathogenesis of Marjolin's ulcer remains to be fully elucidated. The inflammatory irritation hypothesis and immune deficiency hypothesis are the generally accepted 
theories at present (8). However, a recent study has indicated that Marjolin's ulcer mainly exhibit infiltrating growth along the direction of tension. Therefore, tension stimulation is considered a potential factor in the development and progression of Marjolin's ulcer (9).

Marjolin's ulcers have been described by numerous previous studies. However, due to the lack of large-scale studies, the reported aetiology and clinical characteristics, as well as treatment methods and effects vary considerably $(5,10,11)$. For instance, the recurrence times of Marjolin's ulcers vary between studies; certain studies have reported the mean recurrence time of $4.66 \pm 2.07$ months, while others have reported recurrence times of 3-10 months with a mean of 5.4 months, and still others have reported recurrence in 6-11 months with a mean of 8.8 months $(3,12-14)$. To further elucidate the features of this pathology, the present study retrospectively analyzed the aetiology, clinical characteristics and therapeutic efficiency in patients with Marjolin's ulcer who were admitted to and treated at the Institute of Burn Research of Southwest Hospital (third Military Medical University, Chongqing, China) between January 2013 and December 2017. The present study aimed to provide information that may aid in the establishment of clinical practice guidelines for the diagnosis and treatment of Marjolin's ulcer.

\section{Materials and methods}

Data collection. The clinical data of 140 Marjolin's ulcer patients who were admitted to and treated at the Institute of Burn Research, Southwest Hospital, the Third Military Medical University (Chongqing, China) between January 2013 and December 2017 were collected. All of the cases were confirmed by pathological examination (for patients who were hospitalized several times due to recurrence, only data from the first hospitalization were collected). Among these patients, only 65 patients were followed up, and data regarding their recurrent conditions were statistically analyzed. The following factors of all patients were considered in the statistical analysis: i) Demographic data, including gender, age at initial injury or primary disease onset, and age at the onset of Marjolin's ulcer; ii) clinical characteristics, including initial injury or primary disease, latency period, lesion location, lesion type, lesion area and pathological type; iii) presence of bone invasion and lymph node metastasis of tumor cells; and iv) treatment methods and prognosis, mainly including the number of surgeries, surgical methods.

Statistical analyses. Values are expressed as the mean \pm standard deviation. The analyses were performed using SPSS 18.0 statistical software (SPSS, Inc., Chicago, IL, USA). The correlation between age at onset of initial injury or disease and the length of the latency period were examined using Spearman correlation analyses. The presence of bone invasion for different primary lesion locations were examined using chi-square tests or Fisher's exact test. The age at onset between male and female was analyzed using t-test. The time to recurrence of Marjolin's ulcer for different lesion locations was analyzed using the Kaplan-Meier method. $\mathrm{P}<0.05$ was considered to indicate statistical significance.

\section{Results}

Demographic characteristics. The cohort of 140 Marjolin's ulcer patients comprised 88 males (62.9\%) and 52 females (37.1\%), and the male-to-female ratio was $\sim 1.7: 1.0$. The number of patients and the male-to-female ratio exhibited large variations in different years (Fig. 1A). The majority of the patients resided in the local province $(n=81,57.9 \%)$ and $\sim 40 \%$ of the patients $(n=56)$ came from adjacent provinces, including Guizhou (22.2\%), Sichuan (15.7\%) and Hubei $(2.1 \%)$, while only few patients $(n=3,2.1 \%)$ came from more distant provinces. The age at onset of Marjolin's ulcer ranged from 15-85 years, and the mean age at onset was $53.3 \pm 1.2$ years. The age at the onset of Marjolin's ulcer was $54.4 \pm 1.3$ years in males and $51.4 \pm 2.3$ in females, and the difference was not statistically significant $(\mathrm{P}>0.05$; Fig. 1B). In the present study, none of the Marjolin's ulcer patients were aged $<10$ years. Within the patient population, the subgroup with the age range of 40-49 years was largest, followed by the group aged 50-59 years, while the group aged 10-19 years was the smallest. For males, the age range with the largest population was still 40-49 years, while the smallest population was that with the age range of 20-29 years. For females, the group with the age range of 50-59 years was the largest, while the 10-19-year group was the smallest (Fig. 1C).

Histological types and lesion characteristics. The majority of the 140 Marjolin's ulcer patients had squamous cell carcinomas $(n=123,87.9 \%)$, followed by basal cell carcinomas $(n=10,7.1 \%)$, dermatofibrosarcoma protuberans $(n=6,4.3 \%)$ and adenocarcinoma $(n=1,0.7 \%)$. Representative images of lesions are provided in Fig. 2. In three cases of squamous cell carcinoma, the patients had two locations of invasion on the body; in one case of squamous cell carcinoma, the patient had 3 locations of invasion, while all others had one location of invasion. Overall, Marjolin's ulcer mainly occurred on the lower limbs (42.1\%), followed by the head and neck (34.5\%), whereas the perineum had the lowest incidence $(2.1 \%)$. The lesion types in the 6 cases of dermatofibrosarcoma protuberans were all painless masses. The lesion types of other Marjolin's ulcers were infiltrative ulcers $(n=66,49.3 \%)$ or cauliflower-like masses $(n=68,50.7 \%)$; the difference in the incidence between these two types was not large. The lesion areas ranged from $1-625 \mathrm{~cm}^{2}$ and the mean area was $71.3 \pm 7.7 \mathrm{~cm}^{2}$. In the majority of cases, the lesion area was $>10 \mathrm{~cm}^{2}$ and $\leq 50 \mathrm{~cm}^{2}(35.7 \%)$, followed by the $\leq 10 \mathrm{~cm}^{2}(22.9 \%)$ group and the $>50 \mathrm{~cm}^{2}$ and $\leq 100 \mathrm{~cm}^{2}$ group (20.0\%). Only $5 \%$ of the cases had lesion sizes of $>200 \mathrm{~cm}^{2}$ (Table I).

Primary aetiology, age and latency period. Among the patients of the present study, the most common type of initial injury was flame burns, followed by skin masses (defined as original masses located in the epidermis, dermis or hypodermis, with or without pain and accompanying symptoms), trauma, skin ulcerations caused by repeated scratching/friction and scalding (Table II). Among the 140 patients, the age at initial injury or primary disease onset ranged from 1-75 years, and the mean was 24.6 \pm 1.9 years. Most of the initial injuries or diseases occurred at an age of $\leq 10$ years 

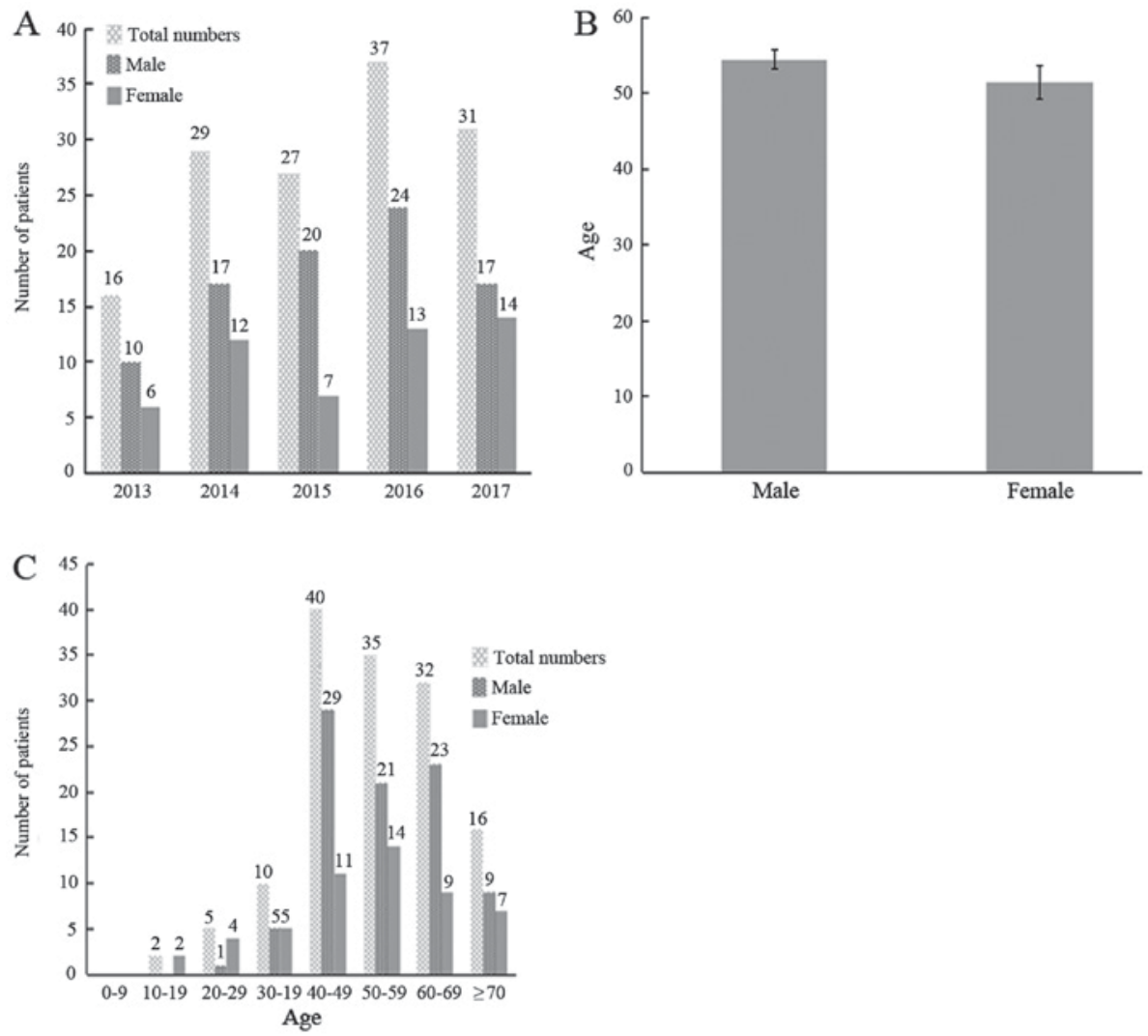

Figure 1. (A) Distribution of Marjolin's ulcer patients in different years and by gender. (B) There was no significant difference in the age at disease onset between male and female Marjolin's ulcer patients ( $\mathrm{P}>0.05)$. (C) Distribution of Marjolin's ulcer patients in different age of onset and by gender. The age is presented in years.
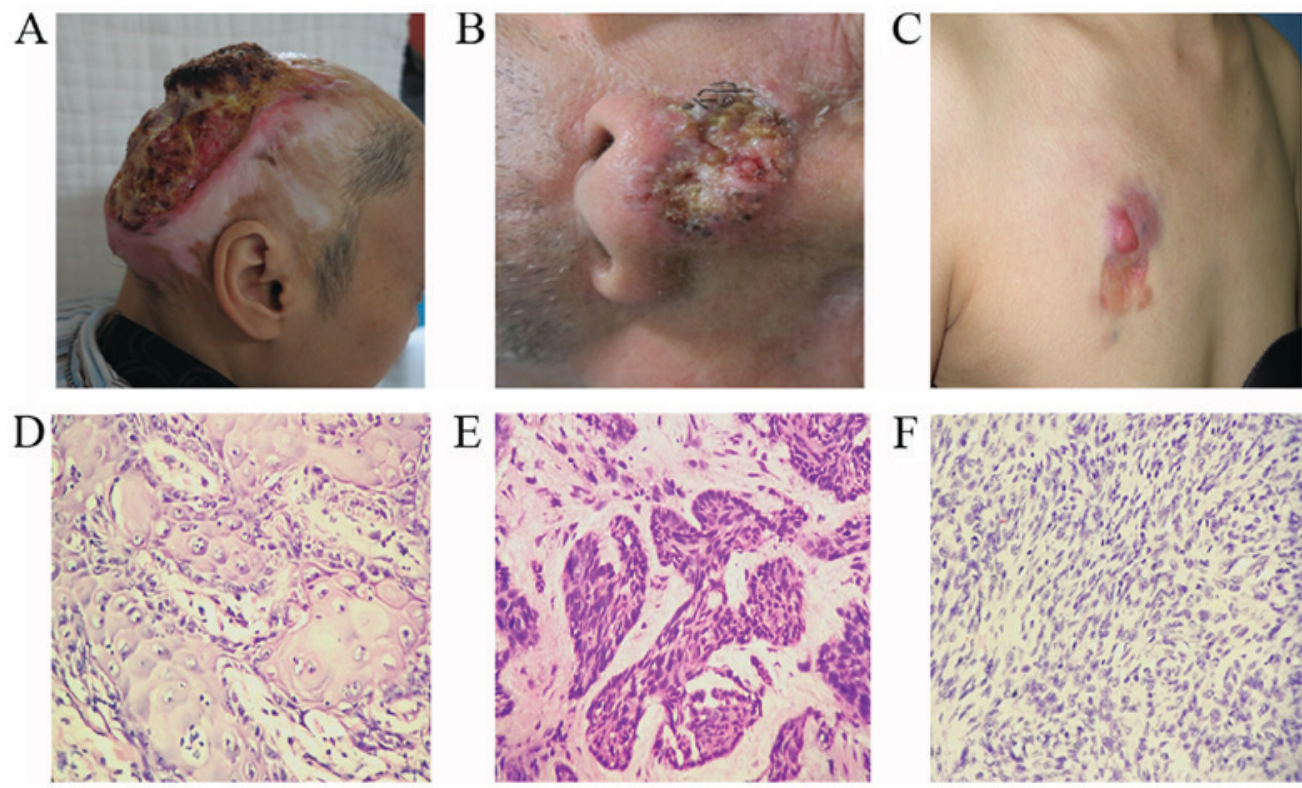

Figure 2. Images and pathological analysis of different histological types of Marjolin's ulcer. Representative images of cases of (A) squamous cell carcinoma, (B) basal cell carcinoma and (C) dermatofibrosarcoma protuberans. Histological images (hematoxylin and eosin staining, x400) of (D) squamous cell carcinoma, (E) basal cell carcinoma and (F) dermatofibrosarcoma protuberans. Images C, D and E are for cases A, B and C, respectively.

(41.4\%), followed by 11-20 years (13.6\%) and 41-50 years (12.9\%; Fig. 3A). Most of the patients had a history of local ulcers after scar formation. The longest latency period was 72 years and the shortest was 2 months, and the mean period was $28.8 \pm 1.7$ years. The majority of the patients had a latency period of $>1$ year $(92.1 \%)$ and only 11 patients $(7.9 \%)$ had a disease onset within 1 year (Table II). The patients' age at onset of initial injury or disease exhibited a significant negative correlation with the length of the latency period $(\mathrm{r}=-0.780, \mathrm{P}<0.01$; Fig. 3B). 
Table I. Distribution of histological types, lesion areas and lesion locations of Marjolin's ulcer.

\begin{tabular}{lc}
\hline Characteristic & N $(\%)$ \\
\hline Histological type & \\
Squamous cell carcinoma & $123(87.9)$ \\
Basal cell carcinoma & $10(7.1)$ \\
Dermatofibrosarcoma protuberans & $6(4.3)$ \\
Adenocarcinoma & $1(0.7)$ \\
Lesion area $\left(\mathrm{cm}^{2}\right)$ & \\
$\leq 10$ & $32(22.9)$ \\
$>10$ and $\leq 50$ & $50(35.7)$ \\
$>50$ and $\leq 100$ & $28(20.0)$ \\
$>100$ and $\leq 200$ & $23(16.4)$ \\
$>200$ & $7(5.0)$ \\
Lesion location & \\
Head, face and neck & $50(35.7)$ \\
Upper limb & $13(9.3)$ \\
Lower limb & $61(43.6)$ \\
Trunk & $10(7.1)$ \\
Perineum & $3(2.1)$ \\
Hip & $8(5.7)$ \\
\hline
\end{tabular}

Table II. Distribution of the causes of initial injury or disease and latency periods of Marjolin's ulcer.

\begin{tabular}{lc}
\hline Characteristic & $\mathrm{N}(\%)$ \\
\hline Initial injury or disease & \\
Flame burn & $63(45.0)$ \\
Skin mass & $22(15.7)$ \\
Trauma & $20(14.3)$ \\
Skin scratch/friction ulceration & $14(10.0)$ \\
Scald & $12(8.6)$ \\
Skin infection & $3(2.1)$ \\
Pigment plaque & $2(1.4)$ \\
Electric burn & $2(1.4)$ \\
Pressure sore & $2(1.4)$ \\
Latency period (years) & \\
$\leq 1$ & $11(7.9)$ \\
$>1$ & $129(92.1)$ \\
\hline
\end{tabular}

Bone invasion of tumors and lymph node metastasis. Among the 140 patients, $46(32.9 \%)$ presented with bone invasion by tumor cells; among them, 38 (27.1\%) only had 1 bone invasion in only 1 location and $8(5.7 \%)$ had 2 bone invasions. Among the 50 cases of Marjolin's ulcer arising from head and neck wounds, 28 had bone invasion, including 25 cases of skull invasion. Among the 13 cases of Marjolin's ulcer arising from upper limb wounds, 2 had bone invasion, and of the 61 cases with lesions from lower limb wounds, 14 had bone invasion. Among the 10 cases with a primary lesion location on the trunk, 1 had bone invasion. Among the 8 cases of Marjolin's ulcer arising from hip wounds, 1 had bone invasion. The percentage of bone invasion in patients with head and neck Marjolin's ulcers was significantly higher than that in patients with other wound locations $\left(\chi^{2}=20.802, \mathrm{P}<0.01\right.$; Table III). Among the 25 patients with skull invasion, 9 had dura invasion, including 4 cases with brain tissue invasion. A total of 33 patients in this group had regional lymph node enlargement diagnosed on palpation, which accounted for $23.6 \%$ of the total cases. Furthermore, 7 patients had lymph node enlargement in 2 locations: The popliteal fossa and the inguen (Table IV). Of the 25 cases with obvious enlargement, those with suspected metastasis based on physical examination underwent lymph node dissection. Only 5 patients $(3.6 \%)$ had lymph node metastasis of tumor cells and the positive rate of dissection was only $20 \%$. Among the 11 patients who had positive positron emission tomography-computed tomography (PET-CT) examination results, pathological examination of lymph nodes revealed tumor cell metastasis in only 2 subjects, and the positive rate was $18.2 \%$. The other patients were all negative (Table V).

Treatment methods. Among the patients included in the present study, 116 received surgical treatment. The remaining $24 \mathrm{did}$ not receive surgery; of these, 14 refused surgery and 10 did not receive surgical treatment due to considerable surgical difficulties and high risks. Among the 116 patients who underwent surgery, 7 received palliative surgery and the other 109 cases received surgery with extended lesion resection. The majority of the patients required only 1 surgery and only few patients required 2 or more surgeries. Depending on the condition of the lesion after resection, 76 patients received skin grafts, 23 received local skin flap repair, 12 underwent amputation and 5 patients underwent island skin flap repair (Table VI).

Prognosis and recurrence. Among the 65 Marjolin's ulcer patients who received follow-up, 22 had recurrence, including 9 cases with recurrence on the head and neck, 3 cases on the upper limbs, 8 cases on the lower limbs and 2 cases on the trunk (data not shown). No significant differences in the recurrence rates of Marjolin's ulcer on different body parts were identified according to the Kaplan-Meier survival analysis (P>0.05; Fig. 4). The shortest recurrence time for Marjolin's ulcer was 1 month and the longest was 11 years; the mean period was $21.7 \pm 7.2$ months. A total of 5 cases (22.7\%) experienced recurrence after $\leq 3$ months, another 5 had a recurrence time of $>3$ months and $\leq 6$ months, another 5 cases had a recurrence time of $>6$ months and $\leq 12$ months, 3 cases $(13.6 \%)$ had a recurrence time of $>1$ year and $\leq 3$ years and 4 cases (18.2\%) had a recurrence time of $>3$ years (Table VII).

\section{Discussion}

The present study reported on 140 cases of Marjolin's ulcer admitted and treated over 5 years, resembling a significantly larger cohort than that reported by other studies $(4,10,15-17)$. The following factors may explain for this high patient number: First, our institution is located in southwest China, a region with a relative low education level of the population where patients do not sufficiently understand the severity of 
Table III. Distribution of Marjolin's ulcer patients with bone invasion in different locations

\begin{tabular}{lcrr}
\hline Tumour location $^{\mathrm{a}}$ & Bone invasion & No invasion & Total \\
\hline Head, face and neck & $28^{\mathrm{b}}$ & 22 & 50 \\
Upper limb & 2 & 11 & 13 \\
Lower limb & 14 & 47 & 61 \\
Trunk & 2 & 3 & 10 \\
Perineum & 0 & 7 & 3 \\
Hip & 1 & 98 & 8 \\
Total & 47 & 145 \\
\hline
\end{tabular}

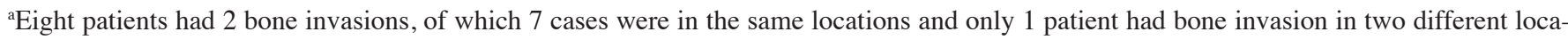
tions. ${ }^{\mathrm{b}} \mathrm{P}<0.05$, compared to the upper limb, lower limb, trunk, perineum and hip groups.
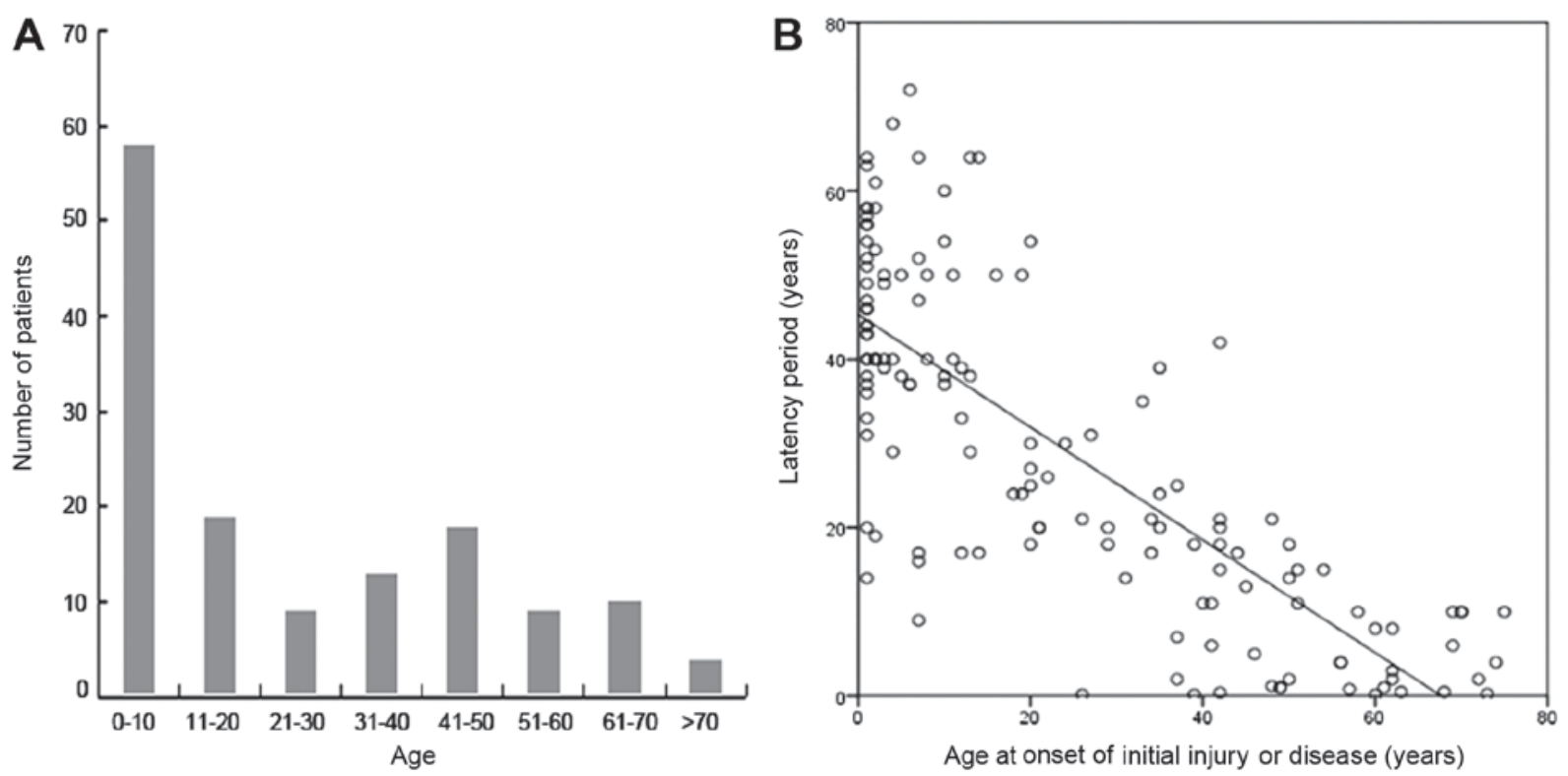

Figure 3. (A) Distribution of age at onset of initial injury or disease in Marjolin's ulcer. (B) The age at onset of initial injury or disease in patients was negatively correlated with the latency period of Marjolin's ulcer $(\mathrm{P}<0.05)$.

Marjolin's ulcer and consequently do not seek timely treatment after scar ulceration. Second, our institution is the largest burn and scar lesion treatment center in southwest China, where a large number of patients from various provinces seek treatment every year. Among these 140 cases, squamous cell carcinoma was the major type of Marjolin's ulcer, followed by basal cell carcinoma and dermatofibrosarcoma protuberans, which was identical to the distribution reported in similar studies $(1,2,5,9)$. However, a typical pathological type, malignant melanoma, which is common in Western countries $(1,6)$, was not observed in the present study. A possible reason may be differences in ethnicity and a lack of long-term sunlight exposure among the population of southwest China. In addition, the male-to-female ratio, age at initial injury and age at the first onset observed in the present study were similar to those in certain previous studies $(2,18)$. Although the initial onset age did not significantly differ between genders, the predilection age of male patients was significantly lower than that of female patients. Furthermore, the present study indicated that the mean latency period for Marjolin's ulcer was 28.8 \pm 1.7 years with a median of 26.5 years, which were shorter than those reported in another study (15). These results indicated that the present study included more patients with shorter latency periods than the previous one. In the present cohort, 11 patients had a latency period of $<1$ year and 3 patients had a latency period of 2 months. Marjolin's ulcer may occur on any part of the body's surface, but they are commonly observed at specific sites. A previous study reported that the lower limbs are the most common site, followed by the head and neck, upper limbs, trunk, hips and perineum (14). The predilection sites of Marjolin's ulcer in the present study were similar to those in other studies. However, Marjolin's ulcers in different locations tended to have different histological types. The histological type of Marjolin's ulcers on the lower limbs and the head and neck was mainly squamous cell carcinoma, while those occurring on the face were mainly basal cell carcinoma and those on the trunk were mostly dermatofibrosarcoma protuberans.

In general, Marjolin's ulcer does not easily metastasize for a considerable amount of time after their onset. The lymphatic system is the primary pathway of metastasis. At 
Table IV. Distribution of patients with lymph node metastasis.

\begin{tabular}{lcc} 
Location & enlargement $(\mathrm{n}=33)(\%)$ & metastasis $(\mathrm{n}=5)(\%)$ \\
\hline Neck & $3(9.1)$ & $2(40)$ \\
Mandible & $1(3.0)$ & 0 \\
Axilla & $5(15.2)$ & $3(60)$ \\
Inguen & $23(69.7)$ & 0 \\
Popliteal fossa & $8(24.2)$ & 0 \\
Inguen and popliteal fossa & $7(21.2)$ &
\end{tabular}

Table V. Accuracy rates of diagnosis of lymph node metastasis using different methods.

\begin{tabular}{lccc}
\hline Detection method & Suspected positive & Pathologically confirmed positive & Positive rate $(\%)$ \\
\hline Clinical diagnosis & 25 & 5 & 20 \\
PET-CT & 11 & 2 & 18.2 \\
\hline
\end{tabular}

Positive rate $(\%)=$ Pathologically confirmed positive/Suspected positive $\mathrm{x} 100 \%$. Clinical diagnosis mainly comprised of the clinical manifestations of enlarged lymph nodes. PET-CT, positron emission tomography-computed tomography.

Table VI. Distribution of the number of surgeries, surgery types and methods for Marjolin's ulcer patients.

\begin{tabular}{lc}
\hline Characteristic & $\mathrm{N}(\%)$ \\
\hline Number of surgeries & \\
0 & $24(17.2)$ \\
1 & $100(71.4)$ \\
2 & $13(9.3)$ \\
3 & $2(1.4)$ \\
4 & $1(0.7)$ \\
Surgical type & \\
Palliative surgery & $7(6.0)$ \\
Extended resection & $109(94.0)$ \\
Surgical method & \\
Skin grafting & $76(65.5)$ \\
Autologous skin grafting & $75(64.6)$ \\
Allogeneic skin grafting & $1(0.9)$ \\
Skin flap & $28(24.1)$ \\
Local skin flap & $23(19.8)$ \\
Island skin flap & $5(4.3)$ \\
Amputation & $12(10.4)$ \\
\hline
\end{tabular}

present, it remains controversial whether preventive lymph node dissection should be performed for Marjolin's ulcer patients (19). It is generally considered that preventive lymph node dissection does not have any obvious influence on tumor recurrence and should not be performed for this purpose (13). However, according to certain studies, the metastatic rate of lymph nodes in the lower limb region is relatively high, and for

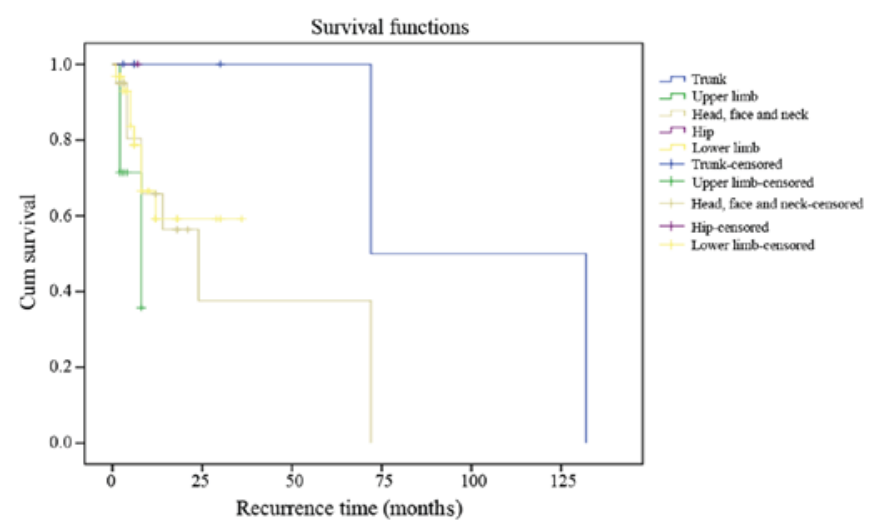

Figure 4. Kaplan-Meier survival curves. The recurrence rates of Marjolin's ulcer in the head and neck, upper limbs, lower limbs and hips exhibited no significant differences. Cum, cumulative.

cases of Marjolin's ulcer without lymph node metastasis in the lower limb region, preventive lymph node dissection should be considered (20). It has also been proposed that the level of tissue differentiation may be used as a basis for determining whether preventive local lymph node dissection should be performed (21). Studies from other countries have reported a rate of metastasis of Marjolin's ulcer in local lymph nodes of $22 \%$ (2). However, a recent study from China indicated that among 187 patients with lymph node enlargement, 64 underwent local lymph node dissection and only 18 developed tumor cell metastasis to the lymph nodes (9). During the treatment of the 140 patients in the present study, only 5 patients developed lymph node metastasis $(3.6 \%)$, yielding a low metastatic rate. In addition, after the surgical dissection of 11 cases of lymph node metastasis diagnosed by PET-CT, only two cases were discovered to be positive, and the positive rate of PET-CT was 
Table VII. Distribution of the time to recurrence of Marjolin's ulcer $(n=22)$.

\begin{tabular}{ll}
\hline Recurrence time (months) & $\mathrm{N}(\%)$ \\
\hline$\leq 3$ & $5(22.7)$ \\
$>3$ and $\leq 6$ & $5(22.7)$ \\
$>6$ and $\leq 12$ & $5(22.7)$ \\
$>12$ and $\leq 36$ & $3(13.6)$ \\
$>36$ & $4(18.2)$ \\
\hline
\end{tabular}

only $18.1 \%$. Therefore, it appears reasonable that the decision to perform regional lymph node dissection should be based on a comprehensive evaluation, including the patient's systemic condition, tumor differentiation level, lesion location, wound size, invasion depth and lymph node imaging results.

At present, the most effective treatment method for Marjolin's ulcer is surgery. Radiotherapy is often used for inoperable patients or as a consolidating treatment after surgery. In the present study, all of the post-operative patients with completely healed wounds were advised to undergo local radiotherapy; however, only a small proportion of the patients chose to do so. In addition, patients who did not undergo surgery were not advised to undergo radiotherapy due to the presence of wounds. Extended lesion resection and amputation were the major surgical methods applied. Extended resection usually includes the areas $>2 \mathrm{~cm}$ away from the ulcer tissues. In certain cases, extension to $5 \mathrm{~cm}$ from the wound edge is recommended (22). The resection depth is determined by the invasion level of the tumor cells, which may reach the superficial layer of the deep fascia, the deep sarcolemma, the muscle tissue or even the periosteum. Deep tissue invasion and/or bone and joint invasion makes the radical resection of the lesion difficult to complete. Radical resection may cause severe impairment of limb function, and wounds cannot be covered after the extensive resection of tumors; the requirement for such extensive resection in Marjolin's ulcer patients is an indicator for amputation (23). However, it is at times difficult to determine whether bone destruction and periosteal reaction detected on imaging result from inflammatory infection or tumor cell invasion. Therefore, bone tissues with suspected involvement may be excised to prevent recurrence caused by residual tumors. The bone invasion locations of Marjolin's ulcer have not been sufficiently evaluated by previous studies. Among the 46 patients with bone invasion in the present study, 25 had skull bone invasion, suggesting that the skull is the bone that is most susceptible to invasion of Marjolin's ulcer. Scalp Marjolin's ulcers that invade the skull are difficult to completely excise and have a high recurrence rate. In addition, as the surgical risk of skull tissue invasion is high, the percentage of patients with skull invasion who refuse surgery is also higher. Among the 25 Marjolin's ulcer patients with skull invasion in the present study, 9 refused surgery and 5 received palliative surgery. Together, these groups represented $56 \%$ of the patients with skull invasion, which is similar to the percentage reported in a previous study (24). After the excision of Marjolin's ulcer, wound repair and functional reconstruction are key to improving life quality. It is generally considered that skin graft repair should be used as much as possible. If bones and tendons are exposed, skin flap repair should be performed. Local skin flap repair should be used if possible, while island skin flap graft repair is the second most desirable alternative. Free skin flap repair should not be the common method of choice. Among the 116 patients who received surgery in the present study, 76 received skin grafts, 28 received local skin flap or island skin flap repair, and none underwent the complex free flap repair. In total, $71.4 \%$ of the patients only required one surgery. These results indicate that skin grafting and simple skin flap repair are still the preferred methods for wound repair after extended resection of Marjolin's ulcer and provide the most satisfactory outcomes.

In the present study, the recurrence rates of Marjolin's ulcers in different locations did not exhibit any significant differences. This may be due to the limited sample size and the number of cases that were lost to follow-up. However, among the 22 cases of recurrence, 17 (77.3\%) were on the head, neck and lower limbs. Furthermore, the trend in recurrence time is another characteristic of Marjolin's ulcer. Various studies have reported that Marjolin's ulcer has a short recurrence time (12-14). However, the present study indicated that the mean recurrence time for Marjolin's ulcers was 21.7 7.2 months, and the recurrence time was significantly longer than that reported in previous studies. One possible reason for this discrepancy is that in the present study, 4 cases had recurrence at $>3$ years after surgery ( 1 case after 11 years and 3 cases after 6 years). Among the cases with recurrence, the majority (68.1\%) relapsed within 1 year after surgery, suggesting that 1 year after surgery is the peak period of recurrence and patients should be closely followed up and observed. Furthermore, the recurrence rates in different body parts had no significant differences in the present study.

In conclusion, the present retrospective study indicated that Marjolin's ulcer mainly occurred in males and was mainly scar carcinoma after a flame burn. The pathological type was mainly squamous cell carcinoma. Autologous skin grafting and local skin flap repair were the major repair methods used. The skull bone was the site most susceptible to invasion.

\section{Acknowledgements}

Not applicable.

\section{Funding}

This study was supported by the National Natural Science Foundation of China (grant no. 81571898) and the New Clinical Technology Plan for Military Medicine and War Trauma Treatment of Southwest Hospital (grant no. SWH2016BZGFKJ-38).

\section{Availability of data and materials}

The datasets used and analyzed during the current study are available from the corresponding author on reasonable request. 


\section{Authors' contributions}

All the authors contributed extensively to the work presented in this study. FX and YSH conceived and designed the study. FX wrote the manuscript. Data analysis and interpretation, revision and final approval of the study were performed by FX, HPS and YSH.

\section{Ethics approval and consent to participate}

This study was approved by the Institutional Review Board of the Southwest Hospital, Third Military Medical University (Chongqing, China).

\section{Patient consent for publication}

The patients whose images are shown in Fig. 2 provided informed consent for the publication of these images.

\section{Competing interests}

The authors declare that they have no competing interests.

\section{References}

1. Onesti MG, Fino P, Fioramonti P, Amorosi V and Scuderi N: Ten years of experience in chronic ulcers and malignant transformation. Int Wound J 12: 447-450, 2015

2. Das KK, Chakaraborty A, Rahman A and Khandkar S: Incidences of malignancy in chronic burn scar ulcers: Experience from Bangladesh. Burns 41: 1315-1321, 2015.

3. Hahn SB, Kim DJ and Jeon CH: Clinical study of Marjolin's ulcer. Yonsei Med J 31: 234-241, 1990.

4. Chalya PL, Mabula JB, Rambau P, Mchembe MD, Kahima KJ, Chandika AB, Giiti G, Masalu N, Ssentongo R and Gilyoma JM: Marjolin's ulcers at a university teaching hospital in Northwestern Tanzania: A retrospective review of 56 cases. World J Surg Oncol 10: 38, 2012.

5. Pekarek B, Buck S and Osher L: A comprehensive review on Marjolin's ulcers. Diagnosis and treatment. J Am Col Certif Wound Spec 3: 60-64, 2011.

6. Bazaliński D, Przybekmita J, Barańska B and Więch P: Marjolin's ulcer in chronic wounds-review of available literature. Contemp Oncol 21: 197-202, 2017.

7. Kheiri B, Osman M and Al Hadidi S: From a burn scar to malignancy! Marjolin's ulcer, a disease of wound neglect. Oxf Med Case Reports 2018: omy044, 2018.
8. Nthumba PM: Marjolin's ulcers: Theories, prognostic factors and their peculiarities in spina bifida patients. World J Surg Oncol 8: $1-5,2010$.

9. Liu Z, Zhou Y, Zhang P, Zhang M, Ren L, Zeng J, Zhou J, Liang P and Huang X: Analysis of clinical characteristics of 187 patients with Marjolin's ulcers. Zhonghua Shao Shang Za Zhi 32: 293-298, 2016 (In Chinese).

10. Sisti A, Pica Alfieri E, Cuomo R, Grimaldi L, Brandi C and Nisi G: Marjolin's ulcer arising in a burn scar. J Burn Care Res 39: 636-639, 2018.

11. Fishman JR and Parker MG: Malignancy and chronic wounds: Marjolin's ulcer. J Burn Care Rehabil 12: 218-23, 1991.

12. Altunay I, Cerman AA, Sakiz D and Ates B: Marjolin's ulcer presenting with in-transit metastases: A case report and literature review. Ann Dermatol 27: 442-445, 2015.

13. Choi JY, Bae YC, Nam SB and Bae SH: Impact of disturbed wound healing after surgery on the prognosis of Marjolin's ulcer. Arc Plast Surg 40: 198-202, 2013.

14. Aydogdu E, Yildirim S and AkSz T: Is surgery an effective and adequate treatment in advanced Marjolin's ulcer? Burns 31: 421-431, 2005.

15. Jellouli-Elloumi A, Kochbati L, Dhraief S, Ben Romdhane K and Maalej M: Cancers arising from burn scars: 62 cases. Ann Dermatol Venereol 130: 413-416, 2003.

16. Fazeli MS, Lebaschi AH, Hajirostam M and Keramati MR: Marjolin's ulcer: Clinical and pathologic features of 83 cases and review of literature. Med J Islam Repub Iran 27: 215-224, 2013.

17. Shen R, Zhang J, Zhang F, DU Y, Liang W, Xu L, DU X, Chen P and Chen X: Clinical characteristics and therapeutic analysis of 51 patients with Marjolin's ulcers. Exp Ther Med 10: 1364-1374, 2015.

18. Yu N, Long X, Lujan-Hernandez JR, Hassan KZ, Bai M, Wang Y, Wang $X$ and Zhao R: Marjolin's ulcer: A preventable malignancy arising from scars. World J Surg Oncol 11: 313, 2013.

19. Fernandes MG, Brandão M and Dias EM: Marjolin's ulcer with axillary lymph node metastasis. Acta Med Port 31: 515, 2018

20. Dupree MT, Boyer JD and Cobb MW: Marjolin's ulcer arising in a burn scar. Cutis 62: 49-51, 1998

21. Ozek C, Celik N, Bilkay U, Akalin T, Erdem O and Cagdas A: Marjolin's ulcer of the scalp: Report of 5 cases and review of the literature. J Burn Care Rehabil 22: 65-69, 2001.

22. Bozkurt M, Kapi E, Kuvat SV and Ozekinci S: Current concepts in the management of Marjolin's ulcers: Outcomes from a standardized treatment protocol in 16 cases. J Burn Care Res 31: 776-780, 2010.

23. Opara KO and Otene IC: Marjolin's ulcers: A review. TNHJ 11: 107-111, 2011.

24. Onah II, Okwor B and Onuigbo WI: Penetrating scalp Marjolin's ulcer involving bone and dura mater in a Nigerian hospital: Case report and literature review. Burns 36: e39-e43, 2010.

(i) (9) This work is licensed under a Creative Commons Attribution-NonCommercial-NoDerivatives 4.0 International (CC BY-NC-ND 4.0) License. 